\title{
Nanocomplexation of thrombin with cationic amylose derivative for improved stability and hemostatic efficacy
}

This article was published in the following Dove Press journal:

International Journal of Nanomedicine

29 January 2015

Number of times this article has been viewed

\author{
Baoxiong Zhuangl,* \\ Zhihua $\mathrm{Li}^{1, *}$ \\ Jiadong Pang,* \\ Wenbin $\mathrm{Li}^{1}$ \\ Pinbo Huang' \\ Jie Wang \\ Yu Zhou' \\ Qing Lin' \\ Quanbo Zhou' \\ Xiao Ye' \\ Huilin Ye \\ Yimin Liu' \\ Li-Ming Zhang ${ }^{2}$ \\ Rufu Chen' \\ 'Department of Hepato-Pancreato-Billiary \\ Surgery, Department of Medical Oncology, \\ Sun Yat-sen Memorial Hospital, Sun Yat-sen \\ University, Guangzhou, People's Republic of \\ China; ${ }^{2}$ DSAPM Lab and PCFM Lab, Institute \\ of Polymer Science, Department of Polymer \\ and Materials Science, School of Chemistry \\ and Chemical Engineering, Sun Yat-sen \\ University, Guangzhou, People's Republic \\ of China
}

*Authors share co-first authorship

Correspondence: Rufu Chen

Department of Hepato-Pancreato-Billiary Surgery, Sun Yat-sen Memorial Hospital, Sun

Yat-sen University, 107 jiang West Road,

Guangzhou 510120, People's Republic of China

Tel +86 I892892828

Email chenrf_sysu@I63.com

Li-Ming Zhang

DSAPM Lab and PCFM Lab, Institute

of Polymer Science, Department of

Polymer and Materials Science, School of

Chemistry and Chemical Engineering, Sun

Yat-sen University, 135 Xingang West Road,

Guangzhou 510275, People's Republic of China

Tel +862084II 2354

Email ceszhlm@mail.sysu.edu.cn
Abstract: As a topical hemostatic agent, thrombin has wide application for many surgical treatments. However, native thrombin always suffers from its physical and chemical instabilities. In this work, a nanocomplexation strategy was developed for modifying the stability and hemostatic efficacy of thrombin, in which a water-soluble cationic amylose derivative containing poly(L-lysine) dendrons was prepared by a click reaction and then used to complex thrombin in an aqueous system. For resultant thrombin nanocomplexes, their morphology and particle size distribution were investigated. Their stabilities were studied in terms of activity retention percentages under different storage time, $\mathrm{pH}$ values, and illumination time. In addition, their ability to achieve in vitro fibrinogen and blood coagulation were evaluated. Via a rat hepatic hemorrhage model and a rat iliac artery hemorrhage model, these thrombin nanocomplexes were confirmed to have good tissue biocompatibility and in vivo hemostatic effectiveness.

Keywords: thrombin, nanoparticles, amylose derivative, complexation, stability, hemostatic activity

\section{Introduction}

Hemorrhage during surgical procedures is common and is potentially life-threatening for patients. ${ }^{1-4}$ In the case of cardiac surgery, for example, bleeding may result from several aspects inherent to cardiac procedures, including the placement of cardiac suture lines in great vessels or chambers of the heart, as well as the creation of high-pressure anastomoses. ${ }^{5}$ Therefore, effective and rapid hemostasis is critical to optimize surgical outcomes. For this reason, various topical hemostatic agents such as thrombin, porcine gelatin, bovine gelatin, bovine collagen, regenerated oxidized cellulose, and their combination products frequently have been used when hemorrhage is not controlled by conventional hemostatic methods. ${ }^{6-9}$ Among them, thrombin is of increasing interest and can be applied to the bleeding site in a dry form or after reconstitution with sterile isotonic saline. ${ }^{9}$ It is known that thrombin is a naturally derived enzyme that has had a prominent role in hemostasis for decades. ${ }^{10,11}$ It can initiate the conversion of fibrinogen to fibrin without a foreign body or inflammatory reactions, and thus has been regarded as an ideal hemostatic agent. ${ }^{10,11}$ However, such a hemostatic agent always suffers from physical and chemical instabilities, which create some difficulties in its formulation and use. In addition, thrombin may rapidly denature and easily lose its biological activity in the environments, with the exception of its physiological one. ${ }^{12}$

In this work, we developed a new route for the stability improvement and bioactivity retention of thrombin. In our strategy, a water-soluble cationic amylose derivative with good biocompatibility was prepared by a click reaction between azidized amylose and 
propargyl focal point poly(L-lysine) dendron, and was then used to complex thrombin in an aqueous system. As a result, thrombin nanocomplexes with good stability and bioactivity retention were obtained. In particular, their hemostatic efficacy was confirmed by fibrinogen binding assay, fresh blood coagulation analysis, rat hepatic hemorrhage model, and rat iliac artery hemorrhage model.

\section{Materials and methods Materials}

Azidized amylose (Amy- $\mathrm{N}_{3}$ ) was prepared according to a similar method reported by Azzam et al. ${ }^{13}$ Propargyl focal point poly(L-lysine) dendron (PLLD-G ${ }_{3}$; generation, 3) was synthesized by divergent and convergent approaches, as reported in a recent publication. ${ }^{14}$ Copper sulfate $\left(\mathrm{CuSO}_{4} \cdot 5 \mathrm{H}_{2} \mathrm{O}\right)$ and sodium ascorbate (99\%) were purchased from Alfa Aesar. Thrombin and chloral hydrate were obtained from Sigma. Sprague-Dawley rats were purchased from the Animal Center of Sun Yat-sen University (Guangzhou, People's Republic of China). Other chemical reagents of analytical grade were obtained commercially and used directly.

\section{Preparation of cationic amylose derivative} For the preparation of water-soluble cationic amylose derivative (CAD) containing poly(L-lysine) dendrons, $10 \mathrm{~mL}$ dimethylsulfoxide, $0.515 \mathrm{~g} \mathrm{PLLD}_{3}(0.29 \mathrm{mmol})$, and $0.05 \mathrm{~g}$ Amy- $\mathrm{N}_{3}$ were added successively under magnetic stirring. After a clear solution was obtained, $18 \mathrm{mg} \mathrm{CuSO} \cdot 5 \mathrm{H}_{2} \mathrm{O}$ and $65 \mathrm{mg}$ sodium ascorbate were then added successively under nitrogen atmosphere. The resultant reaction mixture was heated to $40^{\circ} \mathrm{C}$ for 48 hours. After the reaction, the product was dialyzed in distilled water for 3 days (molecular weight cut-off, 14,000) and lyophilized to obtain CAD with a yield of $65 \%$. Fourier transform infrared (FTIR) measurements were performed to confirm the preparation of CAD by a Perkin-Elmer Paragon 1,000 spectrometer at frequencies ranging from 500 to $4,000 \mathrm{~cm}^{-1}$. Each sample was thoroughly mixed with $\mathrm{KBr}$ and pressed into a pellet form. The content of poly(L-lysine) dendrons in CAD was determined by a Vario EL cube elemental analyzer.

\section{Complexation of CAD with thrombin}

For the complexation of CAD with thrombin, $20 \mathrm{mg}$ CAD was first added into a three-necked flask and dissolved in $500 \mathrm{~mL}$ phosphate-buffered saline solution $(\mathrm{pH} \mathrm{7.4)} \mathrm{at}$ room temperature with gentle stirring. The thrombin stock solution was prepared by dissolving 3,000 $\mathrm{U}$ thrombin in $100 \mathrm{~mL}$ phosphate-buffered saline solution $(\mathrm{pH} \mathrm{7.4)} \mathrm{at}$ room temperature and then was slowly dropped into an aqueous solution of $\mathrm{CAD}$ at room temperature, followed by gentle agitation for $30 \mathrm{~min}$. For resultant $\mathrm{CAD} /$ thrombin complexes, the morphology was observed by a JEM 2010 transmission electron microscopy (Japan), and the particle size distribution was investigated by a ZetaPALS analyzer (Brookhaven Instruments Corporation) at $25^{\circ} \mathrm{C}$ with a $90^{\circ}$ scattering angle.

\section{Stability assays}

For native thrombin and its nanocomplexes with CAD, their stabilities were evaluated in terms of activity retention under different conditions, including storage time $(0,1,2$, $3,4,5,10,20,30,40,50$, and 60 days), $\mathrm{pH}$ values (6.5-6.8, 7.0-7.4, and 7.6-8.0), and illumination time $(0,1,2,3$, 4,5 , and 10 days). To obtain the activity retention, native thrombin or the thrombin nanocomplexes were mixed with the thrombin chromogenic substrate S-2238 (H-D-Phe-PipArg- $p$-nitroanilide, $150 \mu \mathrm{M}$; Chromogenix). After $1 \mathrm{~min}$, the generation of $p$-nitroaniline was monitored by measuring OD at $405 \mathrm{~nm}$. The activity of thrombin was determined as the rate of hydrolysis of S-2238 from the linear range of absorbance at $405 \mathrm{~nm}$ with time.

\section{Fibrinogen coagulation tests}

For the fibrinogen coagulation tests, $1 \mathrm{~mL}$ of $3 \%$ fibrinogen solution and $5 \mathrm{U}$ native thrombin or the thrombin nanocomplexes were added into a test tube $(1.5 \times 5 \mathrm{~cm})$, respectively. The time for the formation of coagulum was recorded to evaluate in vitro hemostatic efficacy of native thrombin or the thrombin nanocomplexes.

\section{Clotting time tests}

For the clotting time tests, $5 \mathrm{U}$ native thrombin or the thrombin nanocomplexes were added, respectively, into a test tube. Then $1 \mathrm{~mL}$ fresh blood was poured into each test tube. The time for the blood coagulation was recorded to evaluate in vitro hemostatic efficacy of native thrombin or the thrombin nanocomplexes.

\section{Tissue biocompatibility evaluation}

For the tissue biocompatibility evaluation, the thrombin nanocomplexes (1 kU/kg mouse) were dispersed homogeneously in distilled water by ultrasonic shaking and then injected into six female BALB/c mice (4 weeks old, $18-20 \mathrm{~g}$ ) through the tail vein. For a comparison, physiological saline was used as a control reagent. After 7 days, all animals were killed. The heart, liver, brain, lung, and 
kidney were separated, washed twice with phosphatebuffered saline solution, and fixed in 4\% formaldehyde for histologic examination. The histologic analyses for these tissues were performed to investigate whether the thrombin nanocomplexes induced tissue damage, inflammation, or systemic thrombosis.

\section{Hemostatic efficacy evaluation in rat hepatic hemorrhage model}

For the hemostatic efficacy evaluation in rat hepatic hemorrhage model, twelve female Sprague-Dawley rats (300-350 g) obtained from the Animal Center of Sun Yat-sen University were randomly assigned to one of two groups and were housed in an air conditioned room (humidity, 72\%-75\%; temperature, $23^{\circ} \mathrm{C}-25^{\circ} \mathrm{C}$; lighting, light-dark cycle every 12 hours). Rats were anesthetized with intraperitoneal injection of $10 \%(\mathrm{w} / \mathrm{v})$ chloral hydrate $(0.3 \mathrm{~mL} / 100 \mathrm{~g})$, shaved on the abdomen, and fixed on their backs to the operating table. The operative area of skin was sterilized with $75 \%$ alcohol, and the abdomen was opened with surgical scissors and forceps, layer by layer, longitudinally. The right lobe of the liver was incised for $1 \times 1$ $\mathrm{cm}$, and the surface tissue was removed to establish a hepatic hemorrhage model, treated, respectively, with native thrombin and the thrombin nanocomplexes.

\section{Hemostatic efficacy evaluation in a rat iliac artery hemorrhage model}

For the hemostatic efficacy evaluation in the rat iliac artery hemorrhage model, twelve female Sprague-Dawley rats (300-350 g) obtained from the Animal Center of Sun Yatsen University were randomly assigned to one of two groups and were housed in an air conditioned room (humidity, $72 \%-75 \%$; temperature, $23^{\circ} \mathrm{C}-25^{\circ} \mathrm{C}$; lighting, light-dark cycle every 12 hours). Rats were anesthetized with intraperitoneal injection of $10 \%(\mathrm{w} / \mathrm{v})$ chloral hydrate $(0.3 \mathrm{~mL} / 100 \mathrm{~g})$, shaved on the abdomen, and fixed on their backs to the operating table. The operative area of skin was sterilized with $75 \%$ alcohol, and the abdomen was opened with surgical scissors and forceps, layer by layer, longitudinally. The right iliac artery was separated from the aorta abdominalis, and the right iliac artery was then punctured with a $1 \mathrm{~mL}$ syringe to establish the right iliac artery hemorrhage model, treated, respectively, with native thrombin and the thrombin nanocomplexes.

\section{Statistical analyses}

The results were expressed as means \pm standard deviations. Differences between means were analyzed for statistical significance by the paired Student's $t$-test and by using SPSS 13.0 statistical analysis software. A value of $P<0.05$ was considered statistically significant.

\section{Results and discussion Characterization of CAD}

The water-soluble CAD containing poly(L-lysine) dendrons was prepared for the first time by a click conjugation reaction between azidized amylose $\left(A m y-N_{3}\right)$ and propargyl focal point poly(L-lysine) dendron of third-generation (PLLD-G ${ }_{3}$ ), as shown in Figure 1. To confirm the preparation of CAD, FTIR analyses were performed. Figure 2 gives the FTIR spectra of Amy-N ${ }_{3}$, PLLD-G$_{3}$, and CAD. As seen, the characteristic absorption bands of Amy- $\mathrm{N}_{3}$ appeared at 3,460 $\mathrm{cm}^{-1}$ ( $\mathrm{v}_{\mathrm{O}-\mathrm{H}}$, pyranose), 2,106 $\mathrm{cm}^{-1}$ (azido groups), and $1,022 \mathrm{~cm}^{-1}\left(\mathrm{v}_{\mathrm{C}-\mathrm{O}}\right.$, pyranose). The characteristic absorption bands of PLLD-G $\mathrm{G}_{3}$ appeared at 2,917 $\mathrm{cm}^{-1}$, $2,842 \mathrm{~cm}^{-1}\left(\mathrm{v}_{\mathrm{C}-\mathrm{H}}\right), 1,604 \mathrm{~cm}^{-1}\left(\mathrm{v}_{\mathrm{C}=\mathrm{O}}\right)$, and $1,396 \mathrm{~cm}^{-1}\left(\mathrm{v}_{\mathrm{CO}-\mathrm{NH}}\right)$. After the click conjugation, the spectrum of CAD did not show the characteristic absorption bands of the azido group $\left(2,106 \mathrm{~cm}^{-1}\right)$ but did exhibit the main characteristic bands of

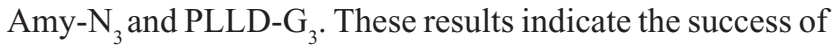
the click conjugation between Amy- $\mathrm{N}_{3}$ and PLLD-G ${ }_{3}$. On the basis of elemental analysis, the degree of substitution of PLLD-G ${ }_{3}$ on the amylose, which is defined as the number of PLLD-G ${ }_{3}$ per 100 anhydroglucose units of amylose, was determined to be 9.35 .

\section{Formation of thrombin nanocomplexes}

Because of the conjugation of cationic poly(L-lysine) dendrons with a high density of primary amino groups, CAD could electrostatically interact with thrombin in an aqueous system, resulting in the formation of CAD/thrombin complexes. To confirm this, we carried out transmission electron microscopy observation and particle size distribution measurements for resultant $\mathrm{CAD} /$ thrombin complexes. As seen from Figure 3, these complexes showed a roughly spherical morphology and had a nanoscale size distribution. Such a similar strategy has been successfully used to obtain colloidally stable nanocomplexes of dendronized cyclodextrin derivatives with plasmid DNA or small interfering RNA for the delivery of nucleic acids. ${ }^{14,15}$

\section{Improved stability of thrombin nanocomplexes}

For the thrombin nanocomplexes with CAD, their stabilities under different conditions were investigated in terms of activity retention when compared with native thrombin, 

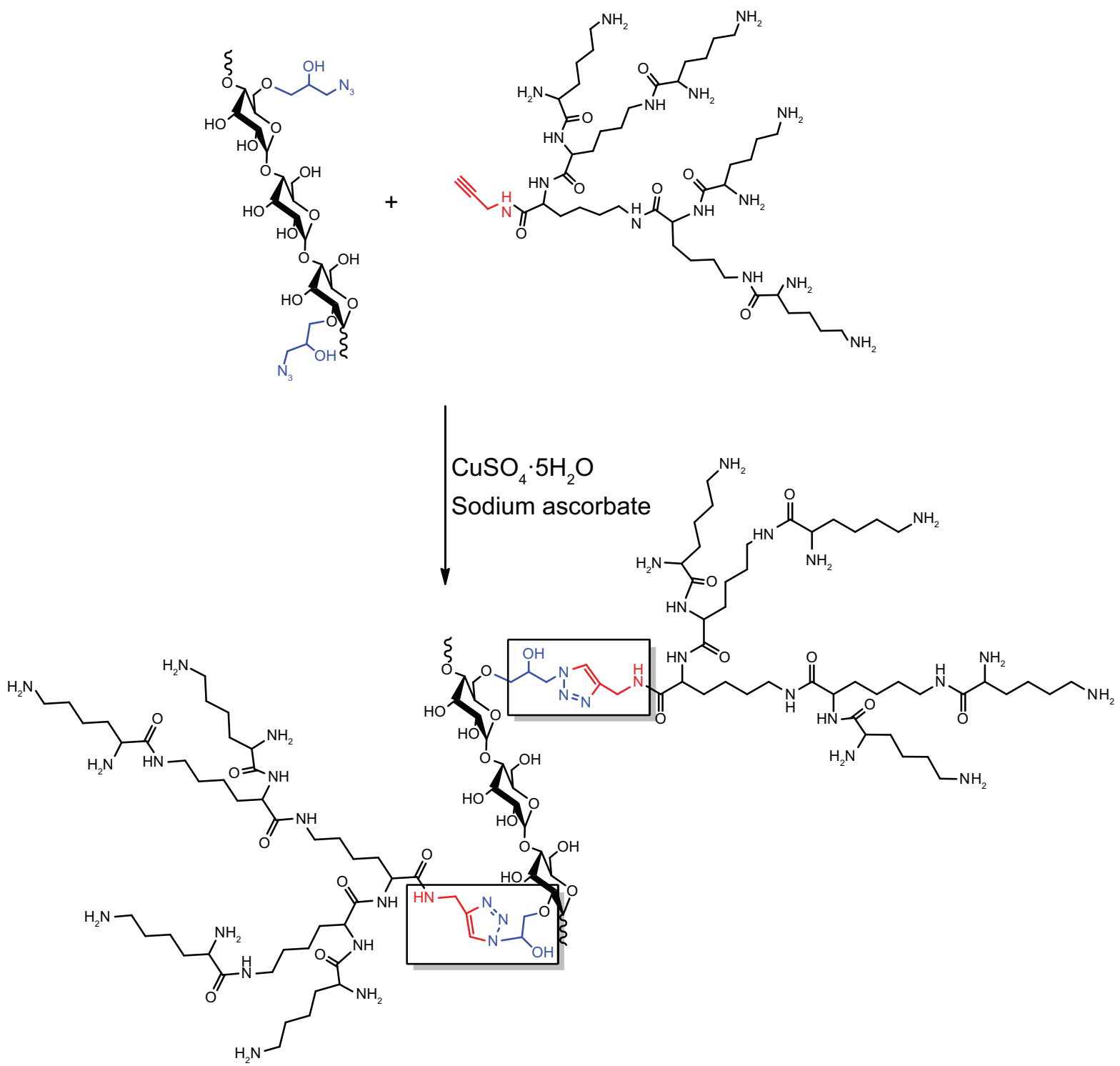

Figure I Schematic illustration for the preparation of cationic amylose derivative (CAD).

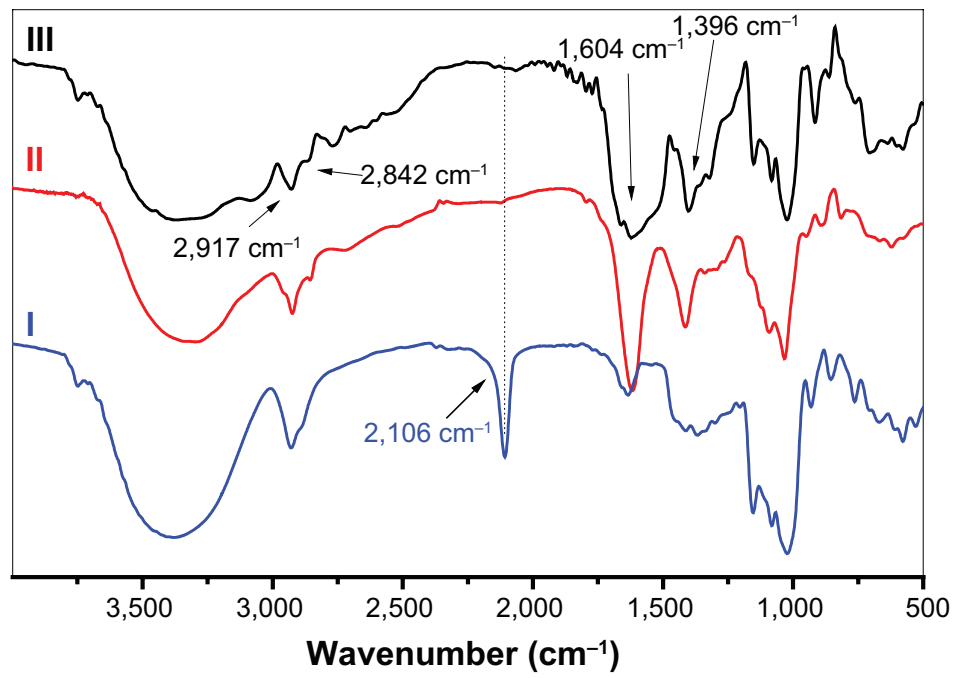

Figure 2 Fourier transform infrared spectra of azidized amylose, propargyl focal point poly(L-lysine) dendron of the third generation and their click conjugate (CAD III). 
A

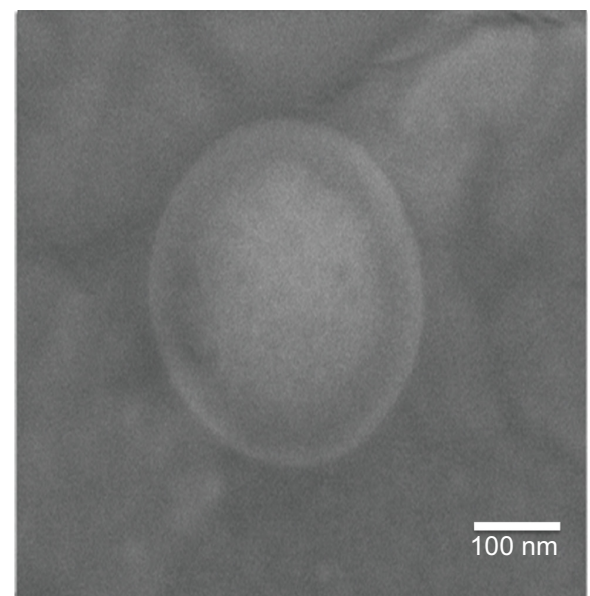

$(\times 10,000)$

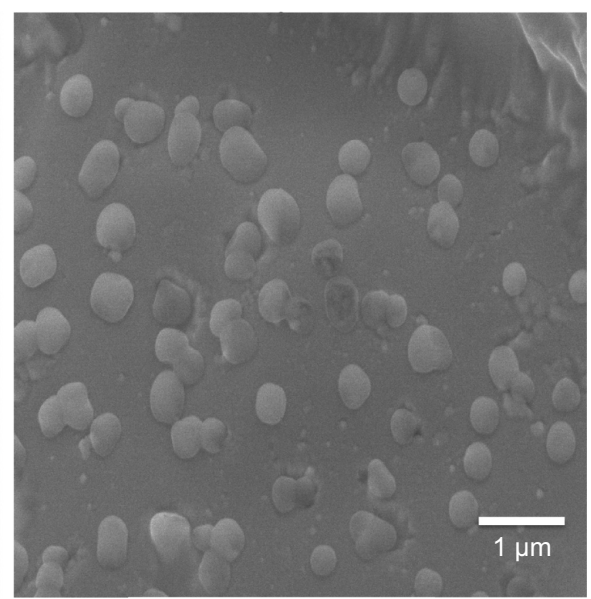

$(\times 2,000)$

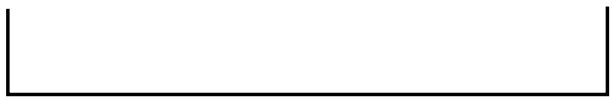

B

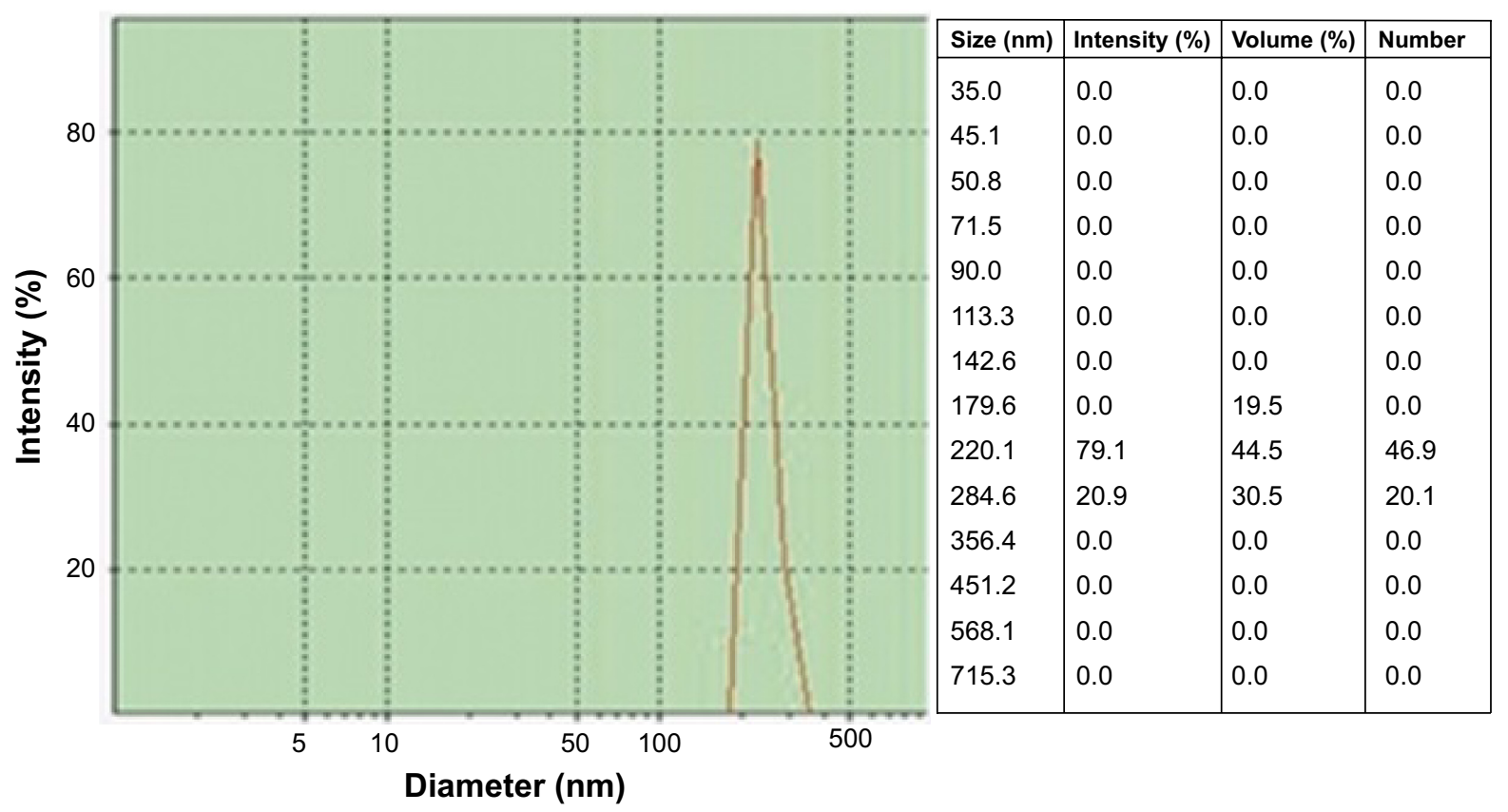

Figure 3 The morphology (A) and particle size distribution (B) of cationic amylose derivative/thrombin complexes.

as shown in Figure 4. To investigate the effect of storage time, the storage time varied from 0 to 60 days, and the storage temperature was kept to $25^{\circ} \mathrm{C}$. From Figure 4A, it was found that the thrombin nanocomplexes could retain a high activity level during a long storage period. In contrast, native thrombin was easy to lose its activity. For example, the activity retention percentage after 5 days was observed to be about $64 \%$ for the thrombin nanocomplexes and about $27 \%$ for native thrombin, respectively. After 60 days, the thrombin nanocomplexes still showed an activity retention percentage higher than $50 \%$, whereas native thrombin only had an activity retention percentage lower than 5\%. Similar phenomena were also observed when we studied the effect of $\mathrm{pH}$ value (Figure 4B) or illumination time (Figure 4C) on the activity retentions of native thrombin and the thrombin nanocomplexes. These results demonstrate that the complexation of CAD with thrombin greatly improves the physical and chemical stabilities of thrombin, which will be favorable for its use as a topical hemostatic agent. 

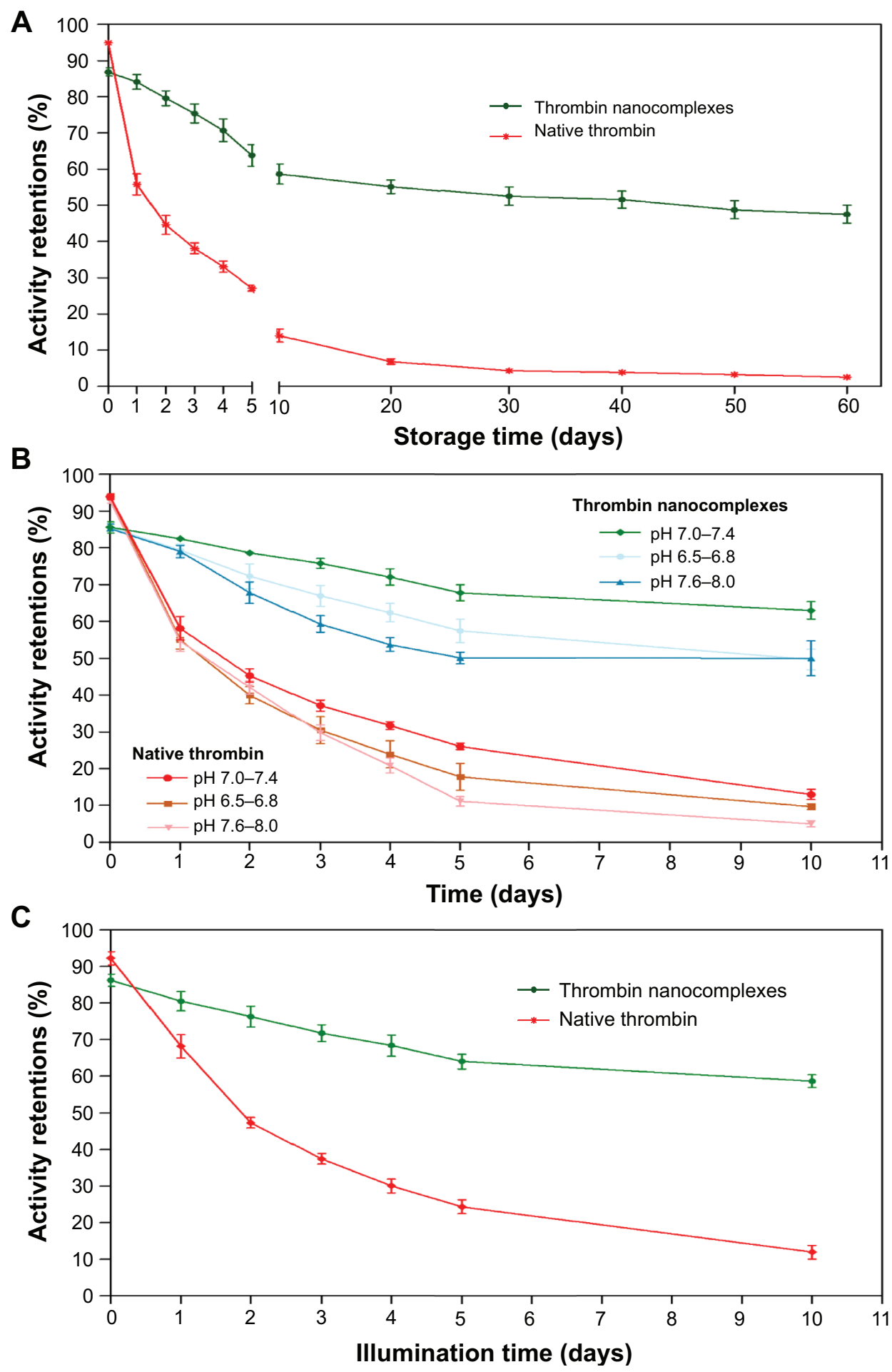

Figure 4 Effects of storage time $(\mathbf{A}), \mathrm{pH}$ value $(\mathbf{B})$, and illumination time $(\mathbf{C})$ on the activity retentions of native thrombin and the thrombin nanocomplexes.

\section{In vitro hemostatic efficacy of thrombin nanocomplexes}

Further investigation dealt with the in vitro hemostatic efficacy of thrombin nanocomplexes by fibrinogen and blood coagulation tests. For a comparison study, native thrombin was also investigated in the same way. As shown in Figure 5, the thrombin nanocomplexes could rapidly induce the fibrinogen and blood coagulations, similar to native thrombin. There are no obvious differences between the fibrinogen coagulation/clotting time of thrombin nanocomplexes and the 
A
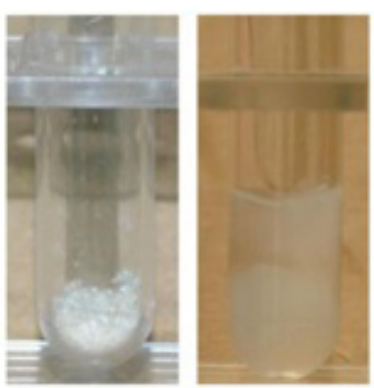

B
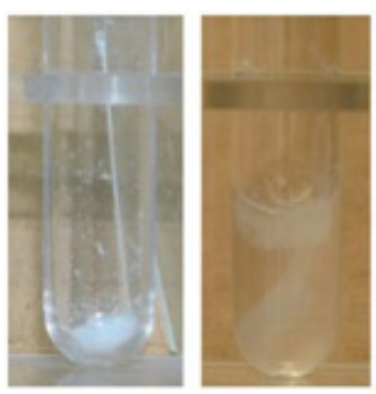

C

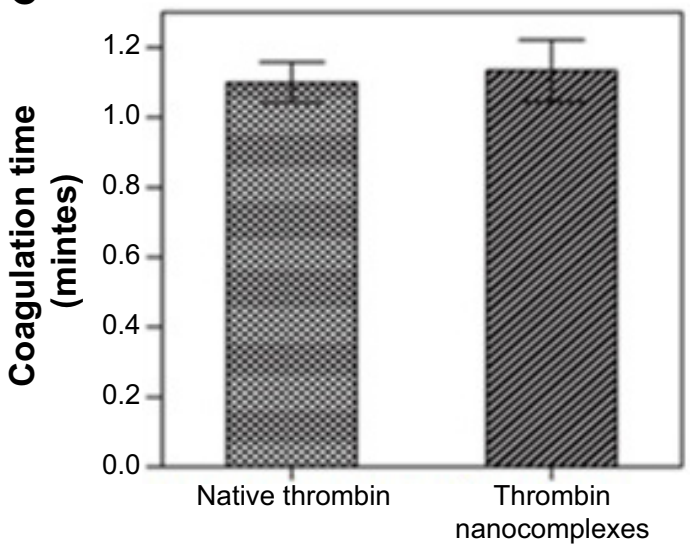

D
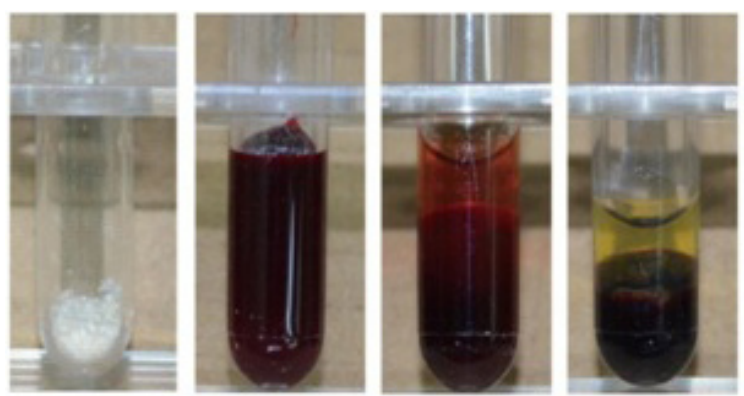

E
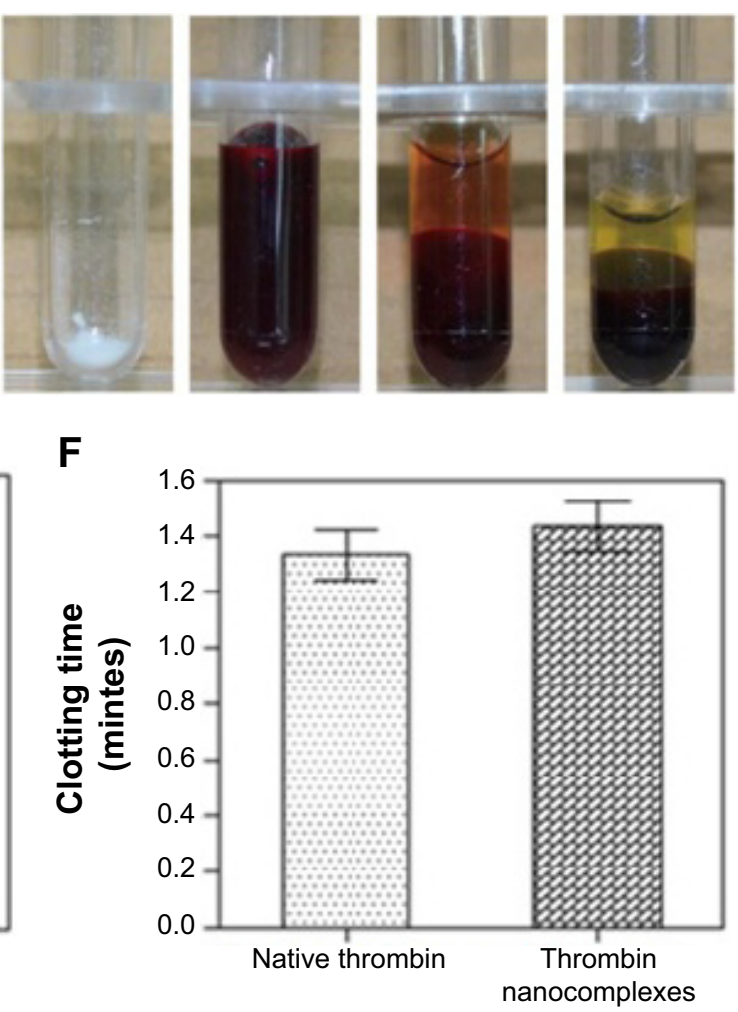

Figure 5 (A) Photographs for the fibrinogen coagulation test in the case of the thrombin nanocomplexes. (B) Photographs for the fibrinogen coagulation test in the case of native thrombin. (C) The fibrinogen coagulation time of native thrombin and the thrombin nanocomplexes. (D) Photographs for the blood coagulation test in the case of the thrombin nanocomplexes. (E) Photographs for the blood coagulation test in the case of native thrombin. (F) The clotting time of native thrombin and the thrombin nanocomplexes.

fibrinogen coagulation/clotting time of native thrombin. The thrombin nanocomplexes still have a strong fibrinogen and blood coagulation ability, similar to native thrombin. These results demonstrate that the nanocomplexation of thrombin with the CAD did not affect significantly the biofunctionality of thrombin.

\section{In vivo tissue biocompatibility and hemostatic efficacy of thrombin nanocomplexes}

To explore the possibility of the thrombin nanocomplexes as an ideal topical hemostatic agent, the in vivo tissue biocompatibility and hemostatic efficacy of thrombin nanocomplexes were investigated. Figure 6 gives the representative histological images of the mice treated, respectively, with physiological saline and aqueous suspension of the thrombin nanocomplexes after 7 days. As seen, the thrombin nanocomplexes did not result in acute inflammation response, thrombus formation, or other adverse effects for the treated heart, liver, brain, lungs, and kidney tissues. Therefore, the thrombin nanocomplexes have a good biocompatibility, which will become an advantage when they are used as a topical hemostatic agent. Figure 7 shows the hemostatic efficacies of thrombin nanocomplexes and native thrombin in rat hepatic hemorrhage model and in rat iliac artery hemorrhage model. As observed, the thrombin nanocomplexes have a good hemostatic property similar to native thrombin, showing its potential application as a topical hemostatic agent. 


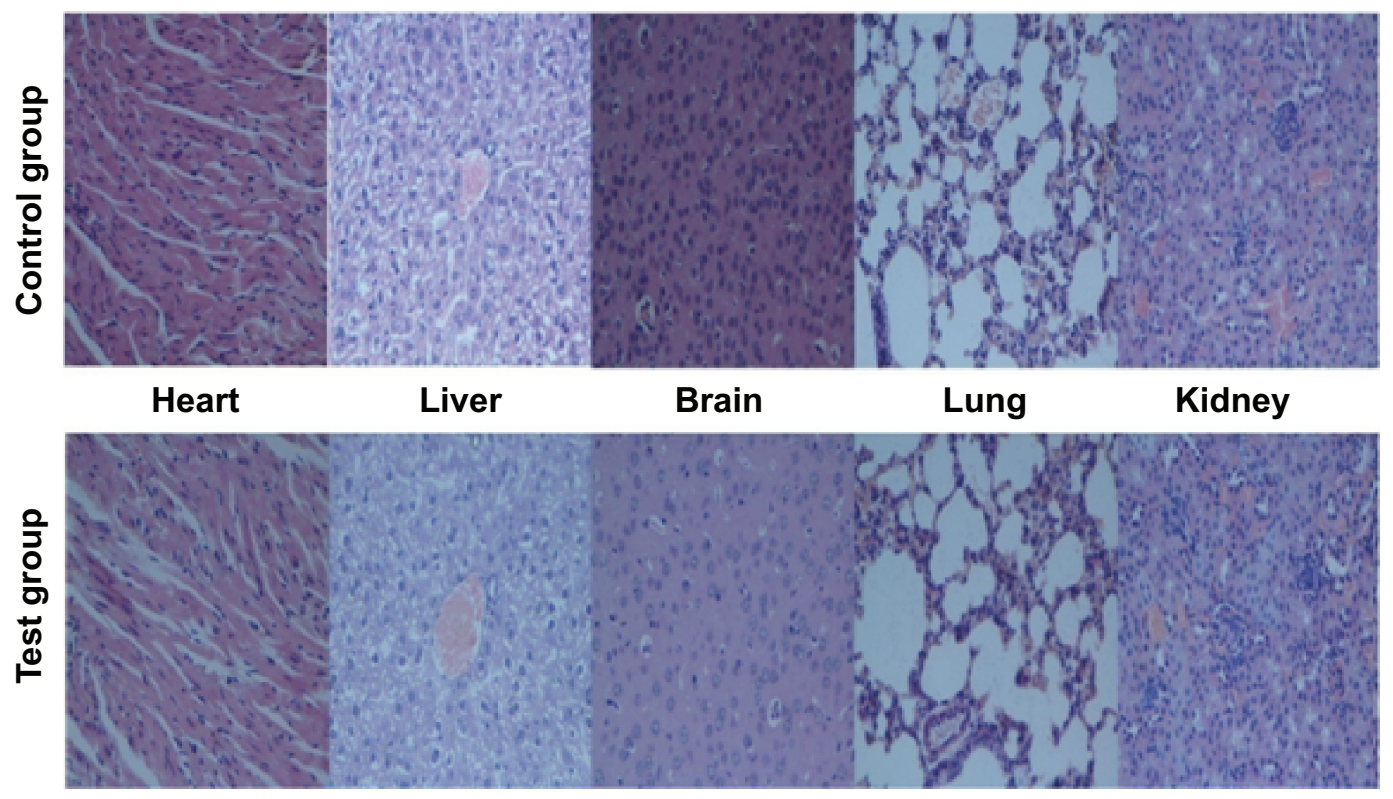

Figure 6 Representative histologic images of the mice treated, respectively, with physiologic saline (control group) and aqueous suspension of the thrombin nanocomplexes (test group) after 7 days.
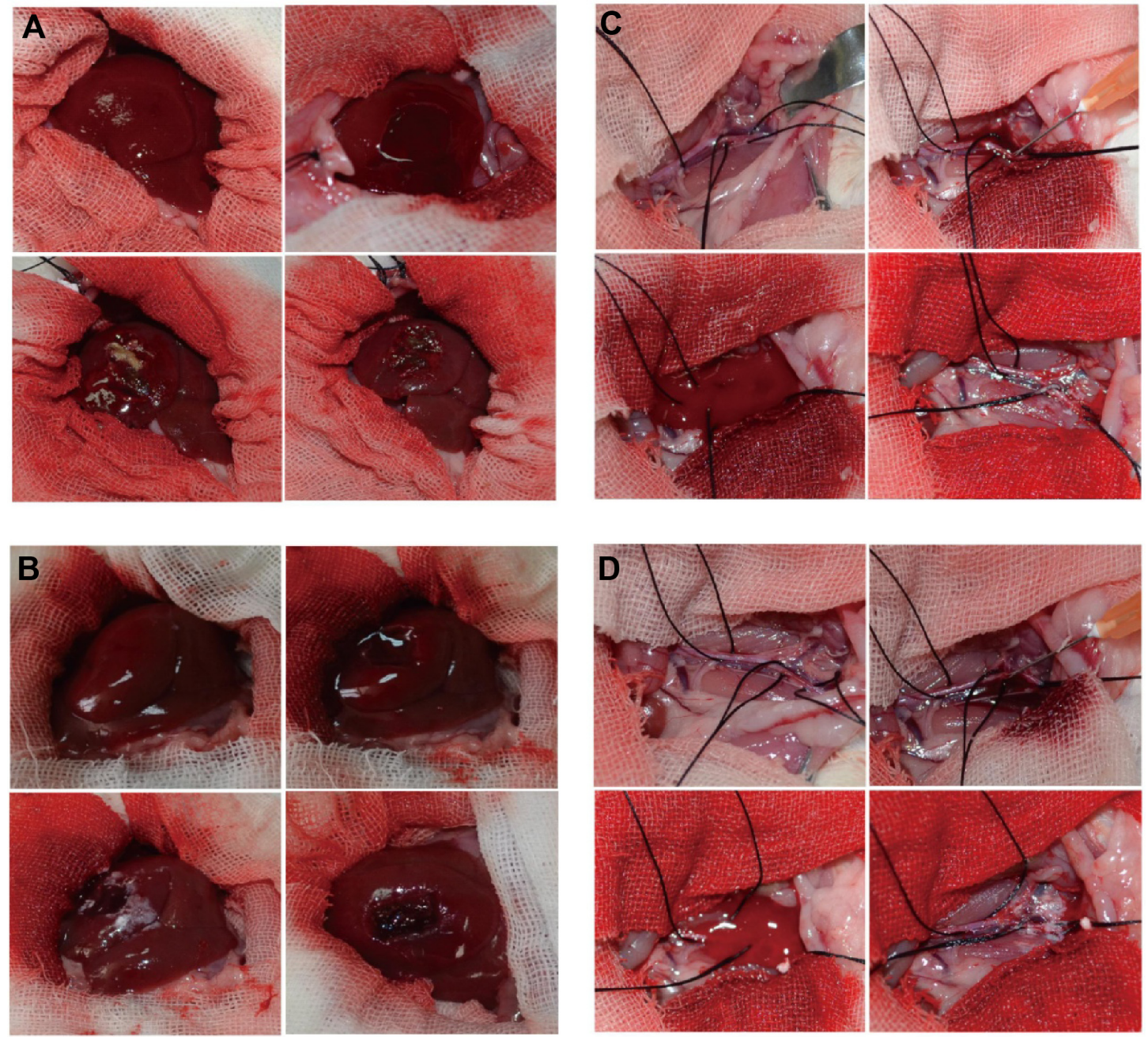

Figure 7 (A) Hemostatic efficacy of thrombin nanocomplexes in rat hepatic hemorrhage model. (B) Hemostatic efficacy of native thrombin in rat hepatic hemorrhage model. (C) Hemostatic efficacy of thrombin nanocomplexes in rat iliac artery hemorrhage model. (D) Hemostatic efficacy of native thrombin in rat iliac artery hemorrhage model. 


\section{Conclusion}

With an attempt to improve the stability and hemostatic properties of thrombin, we developed a nanocomplexation strategy by using a novel cationic amylose derivative containing poly(L-lysine) dendrons with a high density of primary amino groups. The obtained thrombin nanocomplexes were found to have much higher activity retention percentages under different storage times, $\mathrm{pH}$ values, and illumination times when compared with native thrombin. Moreover, they were confirmed to show in vitro and in vivo hemostatic effectiveness, as well as good biocompatibility.

\section{Acknowledgments}

This work was supported by the National Natural Science Foundation of China (51273216, 21074152, J1103305), Doctoral Research Program of Education Ministry in China (20090171110023 and 20130171120095), Integration of Production and Research projects of Guangdong province (20110908), Key Project of Scientific and Technical Innovation for Universities in Guangdong Province (cxzd1102), and Natural Science Foundation of Guangdong Province (S2013010012549 and S2013040014401).

\section{Disclosure}

The authors report no conflicts of interest in this work.

\section{References}

1. Champion HR, Bellamy RF, Roberts CP, Leppaniemi A. A profile of combat injury. J Trauma. 2003;54(5)(suppl):S13-S19.

2. Kauvar DS, Lefering R, Wade CE. Impact of hemorrhage on trauma outcome: an overview of epidemiology, clinical presentations, and therapeutic considerations. J Trauma. 2006;60(6)(suppl): S3-S11.
3. Pusateri AE, McCarthy SJ, Gregory KW, et al. Effect of a chitosanbased hemostatic dressing on blood loss and survival in a model of severe venous hemorrhage and hepatic injury in swine. J Trauma. 2003; 54(1):177-182.

4. Ben-Ari Z, Cardin F, McCormick AP, Wannamethee G, Burroughs AK A predictive model for failure to control bleeding during acute variceal haemorrhage. J Hepatol. 1999;31(3):443-450.

5. Bruckner BA, Blau LN, Rodriguez L, et al. Microporous polysaccharide hemosphere absorbable hemostat use in cardiothoracic surgical procedures. J Cardiothorac Surg. 2014;9(1):134.

6. Gu R, Sun W, Zhou H, et al. The performance of a fly-larva shellderived chitosan sponge as an absorbable surgical hemostatic agent. Biomaterials. 2010;31(6):1270-1277.

7. Balmert SC, Little SR. Biomimetic delivery with micro- and nanoparticles. Adv Mater. 2012;24(28):3757-3778.

8. Watters JM, Van PY, Hamilton GJ, Sambasivan C, Differding JA, Schreiber MA. Advanced hemostatic dressings are not superior to gauze for care under fire scenarios. J Trauma. 2011;70(6):1413-1419.

9. Gabay M. Absorbable hemostatic agents. Am J Health Syst Pharm. 2006;63(13):1244-1253.

10. Achneck HE, Sileshi B, Jamiolkowski RM, Albala DM, Shapiro ML, Lawson JH. A comprehensive review of topical hemostatic agents: efficacy and recommendations for use. Ann Surg. 2010;251(2):217-228.

11. Ofosu FA, Crean S, Reynolds MW. A safety review of topical bovine thrombin-induced generation of antibodies to bovine proteins. Clin Ther. 2009;31(4):679-691.

12. Parrilla JJ, Aznar J, Estelles A. Letter: Fibrinolytic activity in the endometrial adenocarcinoma. Thromb Diath Haemorrh. 1975;34(3): 864-866.

13. Azzam T, Raskin A, Makovitzki A, et al. Cationic polysaccharides for gene delivery. Macromolecules. 2002;35(27):9947-9953.

14. Ma D, Zhang HB, Chen YY, Lin JT, Zhang LM. New cyclodextrin derivative containing poly(L-lysine) dendrons for gene and drug co-delivery. J Colloid Interface Sci. 2013;405:305-311.

15. Li N, Luo HC, Yang C, et al. Cationic star-shaped polymer as an siRNA carrier for reducing MMP-9 expression in skin fibroblast cells and promoting wound healing in diabetic rats. Int J Nanomedicine. 2014;9:3377-3387.
International Journal of Nanomedicine

\section{Publish your work in this journal}

The International Journal of Nanomedicine is an international, peerreviewed journal focusing on the application of nanotechnology in diagnostics, therapeutics, and drug delivery systems throughout the biomedical field. This journal is indexed on PubMed Central, MedLine, CAS, SciSearch ${ }^{\circledR}$, Current Contents ${ }^{\circledR} /$ Clinical Medicine,

\section{Dovepress}

Journal Citation Reports/Science Edition, EMBase, Scopus and the Elsevier Bibliographic databases. The manuscript management system is completely online and includes a very quick and fair peer-review system, which is all easy to use. Visit http://www.dovepress.com/ testimonials.php to read real quotes from published authors. 